\title{
Fermion generations, masses, and mixings in a 6D brane model
}

\author{
Silvestre Aguilar* and Douglas Singleton ${ }^{\dagger}$ \\ Physics Department, CSU Fresno, Fresno, California 93740-8031, USA \\ (Received 21 February 2006; published 18 April 2006)
}

\begin{abstract}
We study the motion of higher dimensional fermions in a nonsingular 6D brane background with an increasing warp factor. This background acts as a potential well trapping fermions and fields of other spins near a $3+1$ dimensional brane. By adjusting the shape of this potential well it is possible to obtain three normalizable zero-mass modes giving a possible higher dimensional solution to the fermion generation puzzle. The three different zero-mass modes correspond to the different angular momentum eigenvalues for rotations around the brane. This bulk angular momentum acts as the family or generation number. The three normalizable zero modes have different profiles with respect to the bulk, thus by coupling the higher dimensional fermion field to a higher dimensional scalar field it is possible to generate both a realistic mass hierarchy and realistic mixings between the different families.
\end{abstract}

DOI: 10.1103/PhysRevD.73.085007

PACS numbers: 11.10.Kk, 04.50.+h, 11.25.Mj

\section{INTRODUCTION}

In the standard model of particle physics [1] there are open questions which have not yet found an answer. Chief among these is the fermion family or generation puzzle as to why the first generation of quarks and leptons (up quark, down quark, electron, and electron neutrino) are replicated in two families or generations of increasing mass (the second generation consisting of charm quark, strange quark, muon, and muon neutrino; the third generation consisting of top quark, bottom quark, tau, and tau neutrino) In addition to explaining why there are heavier copies of the first generation of fermions one would like to explain the mass hierarchy of the generations and the mixings between the generations characterized by the Cabibbo-Kobayashi-Maskawa quark-mixing matrix (CKM). Several ideas have been suggested such as a horizontal family symmetry [2].

Recently theories with extra dimensions have been used in a novel way to try and explain some of the open questions in particle physics and cosmology. In [3-5] the hierarchy problem (i.e. why the gravitational interaction is many orders of magnitude weaker than the strong and electroweak interactions of particle physics) was addressed using large or infinite extra dimensions. Early versions of these extra dimensional models were investigated by several researchers [6-9]. In contrast to extra dimensions in the usual Kaluza-Klein picture, in the models with large or infinite extra dimensions gravity acts in all the spacetime dimensions, while the other particles and fields are confined, up to some energy scale, to a $3+1$ dimensional brane. These recent extra dimensional models have also been applied to answer other questions of particle physics and cosmology. Nonsupersymmetric string models [10] have been constructed which have been able to reproduce the standard model particles from intersecting D5-branes.

\footnotetext{
*Electronic address: sma34@csufresno.edu

${ }^{\dagger}$ Electronic address: dougs@csufresno.edu
}

More phenomenological brane models [11-14] have been constructed to explain the hierarchy of masses and/or the CKM elements of the fermions. In [15,16] brane world models were used to explain dark energy and dark matter. General studies of higher dimensional cosmologies can be found in $[17,18]$.

In this paper we attempt to give a toy model for the generation problem using a brane world model in $6 \mathrm{D}$. We obtain three 4D fermion families from the zero modes of a single 6D spinor field. A mass hierarchy and mixings between the three zero modes are obtained by introducing a Yukawa type interaction between the 6D spinor field and a $6 \mathrm{D}$ scalar field. This gives a common origin (i.e. the higher dimensional Yukawa interaction) for both the mass spectrum and the mixings of the fundamental fermions.

\section{6D GRAVITATIONAL BACKGROUND}

In $[13,19-21]$ a $6 \mathrm{D}$ brane world model was investigated which gave universal gravitational trapping of fields of spins $0, \frac{1}{2}, 1,2$, to the brane. The system considered was $6 \mathrm{D}$ gravity with a cosmological constant and some matter field energy-momentum. The action for this system was

$$
S=\int d^{6} x \sqrt{-6} g\left[\frac{M^{4}}{2}\left({ }^{6} R+2 \Lambda\right)+{ }^{6} L\right]
$$

where $\sqrt{-{ }^{6} g}$ is the determinant, $M$ is the fundamental scale, ${ }^{6} R$ is the scalar curvature, $\Lambda$ is the cosmological constant and ${ }^{6} L$ is the Lagrangian of the matter fields. All of these quantities are six dimensional. The ansatz for the $6 \mathrm{D}$ metric was taken as

$$
d s^{2}=\phi^{2}(r) \eta_{\alpha \beta}\left(x^{\nu}\right) d x^{\alpha} d x^{\beta}-\lambda(r)\left(d r^{2}+r^{2} d \theta^{2}\right),
$$

where the Greek indices $\alpha, \beta, \ldots=0,1,2,3$ refer to 4 dimensional coordinates. The metric of ordinary 4-space, $\eta_{\alpha \beta}\left(x^{\nu}\right)$, has the signature $(+,-,-,-)$. The functions $\phi(r)$ and $\lambda(r)$ depend only on the extra radial coordinate, $r$, and thus are cylindrically symmetric in the transverse 
polar coordinates $(0 \leq r<\infty, 0 \leq \theta<2 \pi)$. The ansatz for the energy-momentum tensor of the matter fields was taken to have the form

$$
T_{\mu \nu}=-g_{\mu \nu} F(r), \quad T_{i j}=-g_{i j} K(r), \quad T_{i \mu}=0 .
$$

Other than satisfying energy-momentum conservation i.e.

$$
\begin{aligned}
\nabla^{A} T_{A B} & =\frac{1}{\sqrt{-{ }^{6} g}} \partial_{A}\left(\sqrt{-{ }^{6} g} T^{A B}\right)+\Gamma_{C D}^{B} T^{C D} \\
& =K^{\prime}+4 \frac{\phi^{\prime}}{\phi}(K-F)=0,
\end{aligned}
$$

the energy-momentum tensor was unrestricted, although it was desirable for it to satisfy physical requirements such as being everywhere finite, and being peaked near the brane. In (4) and in the rest of the paper a prime indicates derivative with respect to $r$.

In $[20,13]$ it was found that the above system had the following nonsingular solution

$$
\phi(r)=\frac{c^{b}+a r^{b}}{c^{b}+r^{b}}
$$

where $a, b, c$ are constants and $a>1$. All other ansatz functions were given in terms of $\phi(r)$.

$$
\lambda(r)=\frac{\rho^{2} \phi^{\prime}}{r}=\rho^{2}(a-1) b c^{b} \frac{r^{b-2}}{\left(c^{b}+r^{b}\right)^{2}},
$$

where $\rho$ is an integration constant with units of length, which was related to the constants $a$ and $b$ by

$$
\frac{\rho^{2} \Lambda}{10 M^{4}}=\frac{b}{a-1} \text {. }
$$

This solution in terms of $\phi(r)$ and $\lambda(r)$ represents a nonsingular, thick brane. The brane thickness is proportional to $c$. Recently the matching conditions for a general thick brane of codimension 2 was given [22]. This provides a general framework in which to study gravitational phenomena and particle trapping in codimension 2 braneworlds. The source functions are also determined by $\phi(r)$.

$$
F(r)=\frac{f_{1}}{2 \phi^{2}}+\frac{3 f_{2}}{4 \phi}, \quad K(r)=\frac{f_{1}}{\phi^{2}}+\frac{f_{2}}{\phi},
$$

where $f_{1}$ and $f_{2}$ are constants given by

$$
f_{1}=-\frac{3 \Lambda}{5} a, \quad f_{2}=\frac{4 \Lambda}{5}(a+1) .
$$

In $[20,13]$ it was shown that for $b=2$ this solution gave a universal, gravitational trapping for fields with spins $0, \frac{1}{2}$, 1,2 within the brane width $(\approx c)$ of $r=0$.

For $b>2$ (6) shows that the scale factor for the extra dimensions, $\lambda(r)=0$ at $r=0$, raising the possibility of having a singularity on the brane and making the solutions with $b>2$ unphysical. However, by looking at invariants such as the Ricci scalar, $R$, one finds that they are nonsingular at $r=0$. This indicates that the zero of $\lambda(r)$ at $r=$ 0 for $b>2$ solutions is not a physical singularity. The Ricci scalar for the above solution is

$$
R=\frac{2 b\left(-5 c^{2 b}+4 a c^{2 b}+10 c^{b} r^{b}-22 a c^{b} r^{b}+10 a^{2} c^{b} r^{b}+4 a r^{2 b}-5 a^{2} r^{2 b}\right)}{(a-1) \rho^{2}\left(c^{b}+a r^{b}\right)^{2}}
$$

It is easy to see that this is finite at $r=0$. Other invariants such as the fully contracted Riemann tensor, $R_{A B}{ }^{C D} R_{C D}{ }^{A B}$, or the square of the Ricci tensor, $R_{A B} R^{A B}$ also turn out to be finite at $r=0$. Thus we take the zero in the scale factor $\lambda(r)$ to be a coordinate rather than physical singularity. Note that $\lambda(r)$ goes to zero both at $r=0$ and $r=\infty$ so that the metric (2) essentially becomes $4 \mathrm{D}$ at these locations. Thus the solution given in (5) is like the 2 brane model of [4] where two 4D branes sandwich the higher dimensional, bulk spacetime.

The weak point in the above is that the source ansatz functions have no clear physical interpreation. It would be desirable to show that an energy-mometum tensor of the general form given by $F(r), K(r)$ could arise from some realistic source such as a scalar field. Because $\phi(r)$ increases as $r \rightarrow \infty(8)$ and (9) indicate that $F(r), K(r)$ have their maximum near $r=0$ and decrease as $\rightarrow \infty$. This behavior is similar to soliton solutions of classical field theory. Thus $F(r), K(r)$ might be considered as modeling some solitonic field configuration which forms the brane. As specific examples Refs. [23,24] investigate solitonic scalar field configurations which form branes. Another possible physical interpretation of the source ansatz functions can be given by transforming the metric given by (2), (5), and (6) via the transformation $r=c \tan ^{2 / b}(\beta / 2)$ and setting $c^{2} / 4=10 M^{4} / \Lambda=\rho^{2}(a-1) / b$. With this the metric takes the form

$$
d s^{2}=\phi^{2}(\beta) \eta_{\alpha \beta}\left(x^{\nu}\right) d x^{\alpha} d x^{\beta}-\frac{c^{2}}{4}\left(d \beta^{2}+\frac{b^{4}}{4} \sin ^{2} \beta d \theta^{2}\right),
$$

where $\phi(\beta)=\frac{1}{2}((a+1)+(1-a) \cos \beta)$. When $b=2$ one can see (after renaming the angles in a standard way as $\beta \rightarrow \theta$ and $\theta \rightarrow \varphi$ ) that the geometry of the extra dimensions is that of a sphere. However when $b>2$ one has zeroes in the $2 D$ scale function, $\lambda(r)$, at $r=0$ and $r=$ $\infty$ and a conical deficit angle of $\delta=2 \pi-b \pi$. This is essentially the "football"-shaped geometry for the extra dimensions considered in [25] (see, in particular, Eq. (24) of [25]). The 6D solution in [25] involved realistic sources: an electromagnetic field in the form of a magnetic flux and 
a bulk cosmological constant. Thus the source ansatz functions, $F(r), K(r)$ may be acting as effective magnetic flux plus bulk cosmological constant.

\section{THREE FAMILIES FROM THREE ZERO MODES}

We now study the motion of a $6 \mathrm{D}$ fermion field in the gravitational background given by (2). We make the identification that different fermion zero-mass modes (i.e. solutions for which the 4D part of the fermion wavefunction satisfies $\left.\gamma_{\mu} \partial^{\mu} \psi\left(x_{\nu}\right)=0\right)$ correspond to different families. Thus we want to see if it is possible to obtain three zero modes. The picture that we present here is a toy model in the sense that the effective 4D fermion fields do not have the full $S U(3) \times S U(2) \times U(1)$ charges of the real standard model fields. As will be shown below the fermions considered here carry only a $U(1)$ charge associated with the rotational symmetry around the brane i.e. the symmetry associated with the extra dimensional variable $\theta$. This is the same as the first example in [12], with the $U(1)$ quantum number being associated with family number. Reference [12] gives other more complex and realistic examples where the fermions carry $U(1) \times U(1)$ or $S U(2) \times U(1)$ charges. In the $U(1) \times U(1)$ example one of the $U(1)$ 's is associated with a horizontal, family symmetry [2] which distinguishes between fermions of different generations, while the other $U(1)$ is then an ordinary gauge symmetry. In the present case the single $U(1)$ is associated with the family symmetry. Other authors [26] also have studied 6D brane models with more realistic standard model charges.

One can gauge [12] any of the above examples using the Kaluza-Klein approach. For example, with the simple $U(1)$ model considered here the metric in (2) has a Killing vector, $\partial_{\theta}$, which via the standard Kaluza-Klein mechanism implies an associated gauge boson in the effective 4D theory. The gauge field arises from the off-diagonal components of the higher dimensional metric as

$$
\begin{aligned}
d s^{2}= & \phi^{2}(r) \eta_{\alpha \beta}\left(x^{\nu}\right) d x^{\alpha} d x^{\beta} \\
& -\lambda(r)\left(d r^{2}+\left(r d \theta+A_{\mu} d x^{\mu}\right)^{2}\right) .
\end{aligned}
$$

This $A_{\mu}$ is analogous to the horizontal gauge boson of the family symmetry models [2]. In [27] it was shown that the zero mode of the gauge field $A_{\mu}$ was localized to the brane. Since horizontal gauge bosons have an experimentally fixed lower mass limit a more realistic model would need to have some symmetry breaking, Higgs mechanism in order to give $A_{\mu}$ an acceptably large mass.

From the previous section we found that in $6 \mathrm{D}$ one has the freedom to let the exponent $b$ take values other than $b=2$. The motion of fermions in the $6 \mathrm{D}$ brane solution of (2) for the $b=2$ case was studied in [21]. It was found that in this case only one zero mode occurred, and thus only one family. Therefore we want to consider the $b>2$ case and show that for a certain range of $b$ it is possible to get three zero modes. The constant $b$ controls the steepness of the scale functions $\phi(r)$ and $\lambda(r)$. Therefore it is reasonable that larger $b$ should give a stronger confinement of the fermions to the vicinity of the brane at $r=0$, and thus to a larger number of zero modes.

The $6 \mathrm{D}$ action and resulting equations of motion for a spinor field are

$$
\begin{aligned}
S_{\Psi} & =\int d^{6} x \sqrt{-{ }^{6} g} \bar{\Psi} i \Gamma^{A} D_{A} \Psi, \\
\Gamma^{A} D_{A} \Psi & =\Gamma^{\mu} D_{\mu} \Psi+\Gamma^{r} D_{r} \Psi+\Gamma^{\theta} D_{\theta} \Psi=0 .
\end{aligned}
$$

In the above $\Gamma^{A}=h_{\bar{B}}^{A} \gamma^{B}$ are the $6 \mathrm{D}$ curved spacetime gamma matrices, and $h_{\bar{B}}^{A}$ are the sechsbiens defined via $g_{A B}=h_{A}^{\bar{A}} h_{B}^{\bar{B}} \eta_{\bar{A} \bar{B}}$. In order to evaluate the 6D Dirac equation in (13) we need to calculated the spin connections

$$
\begin{aligned}
\omega_{M}^{\bar{M} \bar{N}}= & \frac{1}{2} h^{N \bar{M}}\left(\partial_{M} h_{N}^{\bar{N}}-\partial_{N} h_{M}^{\bar{N}}\right)-\frac{1}{2} h^{N \bar{N}}\left(\partial_{M} h_{N}^{\bar{M}}-\partial_{N} h_{M}^{\bar{M}}\right) \\
& -\frac{1}{2} h^{P \bar{M}} h^{Q \bar{N}}\left(\partial_{P} h_{Q \bar{R}}-\partial_{Q} h_{P \bar{R}}\right) h_{M}^{\bar{R}} .
\end{aligned}
$$

The nonzero spin connections are

$$
\omega_{\mu}^{\bar{r} \bar{\nu}}=\delta_{\mu}^{\bar{\nu}} \frac{\sqrt{r \phi^{\prime}}}{\rho}, \quad \omega_{\theta}^{\bar{r} \bar{\theta}}=\sqrt{\frac{r}{\phi^{\prime}}} \partial_{r}\left(\sqrt{r \phi^{\prime}}\right) .
$$

With these one can explicitly calculate the various covariant derivatives in (13)

$$
\begin{gathered}
D_{\mu} \Psi=\left(\partial_{\mu}+\frac{1}{2} \omega_{\mu}^{\bar{r} \bar{\nu}} \gamma_{r} \gamma_{\nu}\right) \Psi, \quad D_{r} \Psi=\partial_{r} \Psi, \\
D_{\theta} \Psi=\left(\partial_{\theta}+\frac{1}{2} \omega_{\theta}^{\bar{r} \bar{\theta}} \gamma_{r} \gamma_{\theta}\right) \Psi .
\end{gathered}
$$

We now assume that the 6D fermion spinor can be decomposed as $\Psi\left(x^{A}\right)=\psi\left(x_{\mu}\right) \otimes \zeta(r, \theta)$ into 4D and 2D parts. We are interested in the zero-mass modes so the 4D fermion part is taken to satisfy $\gamma_{\mu} \partial^{\mu} \psi\left(x_{\nu}\right)=0$. The 2D spinor can be expanded as

$$
\zeta(r, \theta)=\left(\begin{array}{l}
f_{l}(r) \\
g_{l}(r)
\end{array}\right) e^{i l \theta}
$$

We take the gamma matrices of the extra space as in $[12,13]$

$$
\gamma^{r}=\left(\begin{array}{cc}
0 & 1 \\
1 & 0
\end{array}\right) \quad \gamma^{\theta}=\left(\begin{array}{cc}
0 & -i \\
i & 0
\end{array}\right) .
$$

Combining Eqs. (13) -(18) we arrive at the following equations for $f_{l}(r)$ and $g_{l}(r)$

$$
\begin{aligned}
& {\left[\partial_{r}+2 \frac{\phi^{\prime}}{\phi}+\frac{1}{2} \frac{\partial_{r}\left(\sqrt{r \phi^{\prime}}\right)}{\sqrt{r \phi^{\prime}}}+\frac{l}{r}\right] g_{l}(r)=0,} \\
& {\left[\partial_{r}+2 \frac{\phi^{\prime}}{\phi}+\frac{1}{2} \frac{\partial_{r}\left(\sqrt{r \phi^{\prime}}\right)}{\sqrt{r \phi^{\prime}}}-\frac{l}{r}\right] f_{l}(r)=0 .}
\end{aligned}
$$

The solutions for $f_{l}(r)$ and $g_{l}(r)$ are 


$$
\begin{aligned}
& f_{l}(r)=a_{l} \phi(r)^{-2}\left(r \phi^{\prime}(r)\right)^{-(1 / 4)} r^{l}, \\
& g_{l}(r)=b_{l} \phi(r)^{-2}\left(r \phi^{\prime}(r)\right)^{-(1 / 4)} r^{-l} .
\end{aligned}
$$

Because of the $l$ dependence in both $f_{l}(r)$ and $g_{l}(r)$, different $l$ values give fermion fields with different profiles in the bulk. This will result in different masses and mixings for different $l$ values when the fermion field is coupled to a scalar field in the next section. One criteria for the trapping of the fermion field is that it should be normalizable with respect to the extra dimensions $r, \theta$

$$
\begin{aligned}
1 & =\int \sqrt{-{ }^{6} g} \bar{\zeta}(r, \theta) \zeta(r, \theta) d r d \theta \\
& =\int_{0}^{2 \pi} d \theta \int_{0}^{\infty} d r \phi^{4} \rho^{2} \phi^{\prime} \frac{\phi^{-4}}{\sqrt{r \phi^{\prime}}}\left(a_{l}^{2} r^{2 l}+b_{l}^{2} r^{-2 l}\right) \\
& =2 \pi \rho^{2} \int_{0}^{\infty} d r \sqrt{\frac{\phi^{\prime}}{r}}\left(a_{l}^{2} r^{2 l}+b_{l}^{2} r^{-2 l}\right) .
\end{aligned}
$$

From (6) one finds $\sqrt{\phi^{\prime} / r}=(a-$ $1)^{1 / 2} b^{1 / 2} c^{b / 2} r^{(b / 2)-1}\left(c^{b}+r^{b}\right)^{-1}$. Thus in order for (21) to be normalizable and for the particular fermion $l$-mode to be trapped, we want the integral

$$
2 \pi \rho^{2} \sqrt{(a-1) b c^{b}} \int_{0}^{\infty} \frac{r^{ \pm 2 l+(b / 2)-1}}{c^{b}+r^{b}} d r
$$

to be finite. If (22) diverges the particular $l$-mode will not be trapped. This requirement that (22) be finite leads to restrictions on $b$ for particular values of $l$. Evaluating the integral (22) gives

$$
2 \pi^{2} \rho^{2} c^{ \pm 2 l} \sec \left(\frac{2 l \pi}{b}\right) \sqrt{\frac{a-1}{b}} \text { if } \quad b>4|l|,
$$

and (22) diverges if $b \leq 4|l|$. Thus in order to have three normalizable $l$-modes we require that $4<b \leq 8$. Under these conditions the $l=0$, and $|l|=1$ modes are normalized and trapped, while $|l| \geq 2$ modes are not. Since the integrand in (22) is positive definite and only has possible divergences at $r=0$ and $r=\infty$ one can come to this conclusion by investigating the $r \rightarrow 0$ and $r \rightarrow \infty$ behavior of this integrand. For $|l|=1$ one finds that for $l=+1$ the integrand behaves as $\lim _{r \rightarrow 0} \simeq r^{1+(b / 2)}, \lim _{r \rightarrow \infty} \simeq r^{1-(b / 2)}$; for $l=-1$, it behaves as $\lim _{r \rightarrow 0} \simeq r^{-3+(b / 2)}, \lim _{r \rightarrow \infty} \simeq$ $r^{-3-(b / 2)}$. The $r \rightarrow 0$ limit of $l=+1$ and $r \rightarrow \infty$ limit of $l=-1$ give convergent results. On the other hand the $r \rightarrow$ 0 limit of $l=-1$ and $r \rightarrow \infty$ limit of $l=+1$ give convergent results only if $b>4$. One can see the for $b>4$ the $l=0$ mode is normalized. For $|l|=2$ one finds that for $l=+2$ the integrand behaves as $\lim _{r \rightarrow 0} \simeq r^{3+(b / 2)}$, $\lim _{r \rightarrow \infty} \simeq r^{3-(b / 2)} ;$ for $l=-2$, it behaves as $\lim _{r \rightarrow 0} \simeq$ $r^{-5+(b / 2)}, \lim _{r \rightarrow \infty} \simeq r^{-5-(b / 2)}$. The $l=+2$ integral diverges at $r \rightarrow \infty$ and the $l=-2$ diverges at $r \rightarrow 0$ if $b \leq$ 8 . This analysis again shows that one has three normal- izable modes (i.e. three fermion families) when the $4<$ $b \leq 8$.

One could also consider using the criteria for trapping that the fermion action be finite when integrated over the extra dimensions.

$$
\begin{aligned}
S_{\Psi}= & \int d^{6} x \sqrt{-{ }^{6} g} \bar{\Psi} i \Gamma^{A} D_{A} \Psi \\
= & 2 \pi \rho^{2} \int_{0}^{\infty} d r \frac{1}{\phi} \sqrt{\frac{\phi^{\prime}}{r}}\left(a_{l}^{2} r^{2 l}+b_{l}^{2} r^{-2 l}\right) \\
& \times \int d^{4} x \sqrt{-} \bar{\psi} \bar{\psi} i \gamma^{\nu} \partial_{\nu} \psi .
\end{aligned}
$$

The fermions are trapped if the integral over $r$ in the last expression is convergent. This integral is almost the same as the last integral in (21). It differs only by a factor of $1 / \phi$ which comes from the sechsbien that modifies the gamma matrices, $\gamma^{\nu}$. The explicit expression for $\phi^{-1} \sqrt{\phi^{\prime} / r}$ can be read off from (5) and (6). From this one finds that $\phi^{-1} \sqrt{\phi^{\prime} / r} \propto r^{(b / 2)-1}\left(c^{b}+a r^{b}\right)^{-1}$. The only change with respect to the normalization condition (21) is that $r^{b} \rightarrow a r^{b}$ in the denominator. Thus the integral of the action over the extra coordinates will have the same convergence properties as the normalization condition (21), thus giving the same conclusion that three zero-mass modes will be trapped if $4<b \leq 8$.

In [21] only the $b=2$ case in (5) was considered and only one zero-mass mode occurred. Thus the existence of three zero modes is the result of allowing the exponent in (5) to take values $b>2$. In [28] it was shown that the solution of (5), (6), and (8) could be generalized to spacetimes of dimension greater than $6 \mathrm{D}$. For these greater than $6 \mathrm{D}$ spacetimes the exponent, $b$, in (5) was not free, but fixed to $b=2$. This would seem to imply that only in $6 \mathrm{D}$ can one have more than one fermion generation for the background solution given by (5), (6), and (8). However in the case where spacetime greater than $6 \mathrm{D}$ one could consider taking the higher generations as nonzero-mass modes. Also one could try to generalize the other $6 \mathrm{D}$ brane solution given in [21] to spacetime dimensions greater than 6D.

In discussing the masses and mixing between the different families (i.e. different $l$ ) we will need the normalization relationship between $a_{l}$ and $b_{l}$. From (21)-(23) we find

$$
a_{l}^{2} c^{2 l}+b_{l}^{2} c^{-2 l}=\frac{\cos \left(\frac{2 l \pi}{b}\right)}{2 \pi^{2} \rho^{2}} \sqrt{\frac{b}{a-1}} .
$$

This condition allows us to write $b_{l}$ in terms of $a_{l}$ or visa versa.

\section{MIXINGS AND MASSES}

By adjusting the exponent $b$ in our gravitational background solution we have three zero-mass modes which can be taken as a toy model for three generations of fermions. 
There are two problems: first there is no mixing between the different generations due to the orthogonality of the angular parts of the higher dimensional wave functions. Overlap integrals like $\int_{0}^{\infty} \int_{0}^{2 \pi} \bar{\zeta}_{l} \zeta_{m} d r d \theta$, which characterize the mixing between the different states, vanish since $\int_{0}^{2 \pi} e^{-i l \theta} e^{i m \theta} d \theta=0$ if $l \neq m$. Second, all the states are massless, whereas the fermions of the real world have masses that increase with each succeeding family. Following [12] we address both of these issues by introducing a coupling between the $6 D$ fermions and a $6 \mathrm{D}$ scalar field of the form $H_{p}\left(x^{A}\right) \bar{\Psi}_{l}\left(x^{B}\right) \Psi_{l^{\prime}}\left(x^{C}\right)$. This adds to the action a scalar-fermion interaction of the form

$$
S_{s f}=f \int d^{4} x d r d \theta \sqrt{-{ }^{6} g} H_{p} \bar{\Psi}_{l} \Psi_{l^{\prime}}
$$

$f$ is a constant which gives the scalar-fermion coupling strength.

We now take the scalar field to be of the form

$$
H_{p}\left(x^{A}\right)=H_{p}(r) e^{i p \theta},
$$

i.e. only depending on the bulk coordinates $r, \theta$, but not on the brane coordinates $x^{\mu}$. In [12] the same form as in (27) was taken for the scalar field, but certain simplifying assumptions were made about the form of $H(r)$-it was assumed to be either a constant or a delta function. In [14] other forms for the scalar field profile were used. In the following we will determine the form of $H(r)$ by studying the field equations for a scalar field in the background provided by (5) and (6). Note that in the form (27) the scalar field is only dynamical with respect to the extra dimensions, $r, \theta$, but not with respect to the brane spacetime dimensions, $x^{\mu}$. Thus one has a scalar field mechanism for fermion mass generation without a dynamical 4D scalar particle.

Substituting (27) into (26) we find

$$
\begin{aligned}
S_{s f} & =U_{l l^{\prime}} \int d^{4} x \bar{\psi}_{l}\left(x^{\mu}\right) \psi_{l^{\prime}}\left(x^{\mu}\right) \text { where } \\
U_{l l^{\prime}} & =f \int d r d \theta \sqrt{-{ }^{6} g} H_{p}(r) e^{i\left(p-l+l^{\prime}\right) \theta} \bar{\zeta}_{l}(r) \zeta_{l^{\prime}}(r),
\end{aligned}
$$

$U_{l l^{\prime}}$ will be nonzero when $p-l+l^{\prime}=0$. When $l=l^{\prime}$ this gives a mass term and when $l \neq l^{\prime}$ this gives a mixing term between the $l$ and $l^{\prime}$ modes.

To get explicit results for $U_{l l^{\prime}}$ one needs an explicit form for $H_{p}(r)$. This is done by solving the field equations for a test scalar field in the background given by $\phi(r)$ and $\lambda(r)$ for the different $p$-modes. The equation for the scalar field in the background given by (5) and (6) is

$$
\frac{1}{\sqrt{-{ }^{6} g}} \partial_{A}\left(\sqrt{-{ }^{6} g} g^{A B} \partial_{B} H_{p}\left(x^{A}\right)\right)=0 .
$$

Inserting (27) into (29) we get

$$
H_{p}^{\prime \prime}(r)+\left(\frac{1}{r}+\frac{4 \phi^{\prime}(r)}{\phi(r)}\right) H_{p}^{\prime}(r)-\frac{p^{2}}{r^{2}} H_{p}(r)=0 .
$$

From (5) we find that

$$
\frac{4 \phi^{\prime}(r)}{\phi(r)}=\frac{4(a-1) \frac{b}{c}\left(\frac{r}{c}\right)^{b-1}}{\left(1+\left(\frac{r}{c}\right)^{b}\right)\left(1+a\left(\frac{r}{c}\right)^{b}\right)} .
$$

Inserting this expression back into (30) we were not able to find a closed form solution, but were only able to solve it numerically. From the form of $4 \phi^{\prime}(r) / \phi(r)$ in (31) one can see that when $4<b \leq 8$ that the $-p^{2} / r^{2}$ and $1 / r$ terms dominate in the limits $r \rightarrow 0$ and $r \rightarrow \infty$. If one drops the $4 \phi^{\prime}(r) / \phi(r)$ term then one finds that the asymptotic $(r \rightarrow 0$ and $r \rightarrow \infty$ ) solutions to (30) are

$$
\begin{gathered}
H_{p}(r)=A_{+p} r^{|p|} \quad \text { or } \quad A_{-p} r^{-|p|} \quad \text { for } \quad p \neq 0, \\
H_{0}(r)=A_{0} \quad \text { or } \quad B_{0} \ln (r) \quad \text { for } \quad p=0,
\end{gathered}
$$

where $A_{ \pm p}, A_{0}$, and $B_{0}$ constants. These asymptotic solutions gave a fair representation to the numerical solution even for intermediate values of $r$. The $p=0$ solutions can be written in combined form as $H_{0}(r)=B_{0} \ln \left(r / c_{0}\right)$ where $A_{0}=-B_{0} \ln \left(c_{0}\right)$. This form will be used the the next subsection to give masses to the three zero modes. The singularities in $H_{0}(r)$, at $r=0$ and $r=\infty$, are not a problem since the combination of the fermion "wave function" and the metric ansatz functions go to zero fast enough at $r=0$ and $r=\infty$ to negate these singularities in the Yukawa coupling integral (28).

\section{A. Masses}

From (28) one can sees that the mass terms are those for which $l=l^{\prime}$ and thus we want to consider couplings to the $p=0$ scalar mode in (32). We will use the combined form of the two solutions namely $H_{0}(r)=B_{0} \ln \left(r / c_{0}\right)$. With this (28) becomes

$$
\begin{aligned}
m_{l}= & U_{l l}=f \int d r d \theta \sqrt{-{ }^{6} g} H_{0}(r) \bar{\zeta}_{l}(r) \zeta_{l}(r) \\
= & 2 f B_{0} \pi \rho^{2} \sqrt{(a-1) b c^{b}} \int_{0}^{\infty} \frac{\ln \left(\frac{r}{c_{0}}\right) r^{b / 2-1}}{c^{b}+r^{b}} \\
& \times\left[a_{l}^{2} r^{2 l}+b_{l}^{2} r^{-2 l}\right] d r \\
= & f B_{0}\left(\ln \left(\frac{c}{c_{0}}\right)+\frac{\pi K_{l}}{b} \tan \left(\frac{2 l \pi}{b}\right)\right) \text { where } \\
K_{l}= & \frac{4 a_{l}^{2} c^{2 l} \pi^{2} \rho^{2}}{\cos \left(\frac{2 \pi l}{b}\right)} \sqrt{\frac{a-1}{b}}-1 .
\end{aligned}
$$

In arriving at the final line in (34) where have used (25) to replace $b_{l}$ in terms of $a_{l}$. Looking at only the $\tan (2 l \pi / b)$ term and taking $f B_{0} K_{l} \pi / b>0$ gives a hierarchy of masses of $m_{-1}<m_{0}<m_{+1}$. However $m_{-1}<0$ and $m_{0}=$ 0 which is phenomenologically wrong. Taking $\ln \left(c / c_{0}\right)$ as positive (i.e. $c>c_{0}$ ) and sufficently large can shift the 
mass spectrum so that all masses are positive and while still maintaining the hierarchy $m_{-1}<m_{0}<m_{+1}$. Because our fermions only carry a $U(1)$ charge the above hierarchy is a toy model. Here, as an example, we take the three fermions as the "down" sector of quark generations where $l=-1$ is the down quark, $l=0$ is the strange quark, and $l=+1$ is the bottom quark. Taking the masses of the down, strange and bottom quarks as $m_{-1}=m_{d}=4 \mathrm{MeV}, m_{0}=m_{s}=$ $100 \mathrm{MeV}$ and $m_{+1}=m_{b}=4400 \mathrm{MeV}$ we find from (34)

$$
\begin{aligned}
& \frac{m_{d}}{m_{s}}=\frac{m_{-1}}{m_{0}}=0.04=\left(\frac{-K_{-1} \pi \tan (2 \pi / b)}{b \ln \left(c / c_{0}\right)}+1\right), \\
& \frac{m_{b}}{m_{s}}=\frac{m_{+1}}{m_{0}}=44.00=\left(\frac{K_{+1} \pi \tan (2 \pi / b)}{b \ln \left(c / c_{0}\right)}+1\right) .
\end{aligned}
$$

Solving these equations for $a_{1}$ and $a_{-1}$ (which are embedded in the definition of $K_{+1}$ and $K_{-1}$ ) gives

$$
\begin{aligned}
a_{1} & =\frac{D}{c^{2}} \sqrt{(1+43 x) \cos \left(\frac{2 \pi}{b}\right)}, \\
a_{-1} & =D \sqrt{(1+0.96 x) \cos \left(\frac{2 \pi}{b}\right)},
\end{aligned}
$$

where $x=\frac{b \ln \left(c / c_{0}\right)}{\pi \tan (2 \pi / b)}$ and $D^{2}=\frac{c^{2}}{4 \pi^{2} \rho^{2}}\left(\frac{b}{a-1}\right)^{1 / 2}$. If $a_{1}$ and $a_{-1}$ are chosen as in (36) then the mass ratios in (35) are obtained.

\section{B. Mixings}

A similar analysis can be carried out with the mixings between the different "families" characterized by different $l$ number. The mixings are delineated by $U_{0,1}, U_{1,0}, U_{1,-1}$, $U_{-1,1}, U_{0,-1}$, and $U_{-1,0}$. In the case of mixings the scalar field must have a nonzero angular eigenvalue (i.e. $H_{p}(r, \theta)=H_{p}(r) e^{i p \theta}$ with $\left.p \neq 0\right)$ which satisfies $p-l+$ $l^{\prime}=0$. Thus for $U_{-1,0}$ and $U_{0,1}$ one needs $p=-1$; for $U_{1,0}$ and $U_{0,-1}$ one needs $p=1$; for $U_{1,-1}$ one needs $p=2$; for $U_{-1,1}$ one needs $p=-2$. We will require the following relationship between the mixings: $U_{0,1}=U_{1,0}, U_{1,-1}=$ $U_{-1,1}$ and $U_{0,-1}=U_{-1,0}$. This in turn implies that $H_{p}(r)$ should depend only on $|p|$ (e.g. $H_{1}(r)=H_{-1}(r)$ ). Looking at the first line in (32) this means that we can take either the first solution or the second but not the sum in general unless $A_{+1}=A_{-1}$. In what follows we will take $H_{p}=$ $A_{+p} r^{|p|}$. The conclusions are not qualitatively different if we make the other choice $H_{p}=A_{-p} r^{-|p|}$. With this we find

$$
\begin{aligned}
U_{l l^{\prime}}= & f \int d r d \theta \sqrt{-6} g H_{p}(r) \bar{\zeta}_{l}(r) \zeta_{l^{\prime}}(r) \\
= & 2 \pi \rho^{2} f \sqrt{(a-1) b c^{b}} \int_{0}^{\infty} H_{p}(r) \frac{r^{(b / 2)-1}}{c^{b}+r^{b}} \\
& \times\left[a_{l}^{*} a_{l^{\prime}} r^{l+l^{\prime}}+b_{l}^{*} b_{l^{\prime}} r^{-l-l^{\prime}}\right],
\end{aligned}
$$

where in (37) we have carried out the $d \theta$ integration, and the condition $p-l+l^{\prime}$ holds. Inserting $H_{p}(r)=A_{+p} r^{|p|}$ in (37) and assuming all $a_{l}$ and $b_{l}$ are purely real we get

$$
\begin{aligned}
& U_{l l^{\prime}}=2 \pi \rho^{2} f \sqrt{(a-1) b c^{b}} A_{+p} \int_{0}^{\infty} \frac{r^{(b / 2)+p-1}}{c^{b}+r^{b}} \\
& \times\left[a_{l} a_{l^{\prime}} r^{l+l^{\prime}}+b_{l} b_{l^{\prime}} r^{-l-l^{\prime}}\right] \\
& =2 \pi^{2} \rho^{2} f \sqrt{\frac{a-1}{b}} A_{+p} c^{|p|}\left[a_{l} a_{l^{\prime}} c^{l+l^{\prime}} \sec \left(\frac{\left(l+l^{\prime}+|p|\right) \pi}{b}\right)\right. \\
& \left.+b_{l} b_{l^{\prime}} c^{-l-l^{\prime}} \sec \left(\frac{\left(l+l^{\prime}-|p|\right) \pi}{b}\right)\right] \text {. }
\end{aligned}
$$

Now the four cases $\left(l=0, l^{\prime}=-1\right),\left(l=-1, l^{\prime}=0\right)$, $\left(l=0, l^{\prime}=1\right)$ and $\left(l=1, l^{\prime}=0\right)$ involve $|p|=1$ and from (38) yield

$$
\begin{gathered}
U_{0,-1}=U_{-1,0}=\frac{f D A_{+1} c^{2}}{2}\left(\frac{a_{0} a_{-1}}{c^{2}}+b_{0} b_{-1} \sec \left(\frac{2 \pi}{b}\right)\right), \\
U_{0,1}=U_{1,0}=\frac{f D A_{+1} c^{2}}{2}\left(\sec \left(\frac{2 \pi}{b}\right) a_{0} a_{-1}+\frac{b_{0} b_{-1}}{c^{2}}\right) .
\end{gathered}
$$

For the two cases $l=1, l^{\prime}=-1, l=-1, l^{\prime}=1$ one has $|p|=2$

$$
U_{1,-1}=U_{-1,1}=\frac{f D A_{+2} c^{2}}{2}\left(a_{1} a_{-1}+b_{1} b_{-1}\right) \sec \left(\frac{2 \pi}{b}\right),
$$

where as in the previous subsection $D^{2}=\frac{c^{2}}{4 \pi^{2} \rho^{2}}\left(\frac{b}{a-1}\right)^{1 / 2}$. We now show that parameters (i.e. $a, b, c, a_{0}$ ) can be chosen so the ratios of the above mixings match the ratios of the CKM mixing matrix elements. If this can be done then taking $a_{ \pm 1}$ as in (36) will yield the correct mass ratios. From [29] we take $U_{0,-1}=V_{u s} \approx 0.224, U_{0,1}=V_{c b} \approx$ 0.040 and $U_{-1,1}=V_{u b} \approx 0.0036$. Again note that since in our model we only have one flavor in each family (here taken as the down flavor or sector) these associations between $U_{i, j}$ and $V_{i j}$ are to be taken as representing generic interfamily mixing. Now combining (39)-(41) gives

$$
\begin{gathered}
\frac{U_{0,-1}}{U_{0,1}}=\frac{a_{0} a_{-1}+c^{2} \sec \left(\frac{2 \pi}{b}\right) b_{0} b_{-1}}{b_{0} b_{1}+c^{2} \sec \left(\frac{2 \pi}{b}\right) a_{0} a_{1}} \approx 5.6, \\
\frac{U_{0,-1}}{U_{-1,1}}=\frac{A_{+1}\left(\frac{a_{0} a_{-1}}{c^{2}}+b_{0} b_{-1} \sec \left(\frac{2 \pi}{b}\right)\right)}{A_{+2}\left(a_{1} a_{-1}+b_{1} b_{-1}\right) \sec \left(\frac{2 \pi}{b}\right)} \approx 60.5 .
\end{gathered}
$$

To determine the last ratio one needs to determine the normalization constants, $A_{+1}$ and $A_{+2}$ of the $p=1$ and $p=2$ scalar field modes. For this one needs to explicitly evaluate the integral $1=A_{p}^{2} \int \sqrt{{ }^{6} g} H_{p}^{2} d r d \theta$ for $p=1$ and $p=2$, with $H_{p}(r)$ given by (32). Explicitly the ratio $A_{+1} / A_{+2}$ is 


$$
\frac{A_{+1}}{A_{+2}}=c \sqrt{\frac{\int_{0}^{\infty} \frac{\left(1+a y^{b}\right)^{4}}{\left(1+y^{b}\right)^{6}} y^{b+3} d y}{\int_{0}^{\infty} \frac{\left(1+a y^{b}\right)^{4}}{\left(1+y^{b}\right)^{6}} y^{b+1} d y}} .
$$

The two mass ratios have already been fixed by choosing $a_{ \pm 1}$ [this also fixes $b_{ \pm 1}$ because of (25)] as in (36). Next the mixing ratio, $\frac{U_{0,-1}}{U_{0,1}}$, in (42) can be fixed by choosing $a_{0}$ [this also fixes $b_{0}$ because of (25)]. In particular let us parameterize $a_{0}$ as $a_{0}=\frac{D}{c} \eta$ so that $b_{0}=\frac{D}{c} \sqrt{2-\eta^{2}}$ where $\eta$ is arbitrary, and $D$ and $c$ are previously defined. In this way one can see that (42) and also (36) are independent of $c$. Finally one can fix (43) by choosing $a$ in (44) to give the ratio $A_{+1} / A_{+2}$. As an example choose $a=7.5, b=4.041$, $c=1.0, D=1.0$ (this can be done by adjusting $\rho$ ), and $x=0.001$ (this can be done by adjusting $c_{0}$ ). In this way one finds $a_{1} \approx 0.1289$ and $a_{-1} \approx 0.1263$. The associated $b_{l}$ 's are $b_{1} \approx 0.1234$ and $b_{-1} \approx 0.1262$. Using these one find that the mass ratios in (35) are satisfied. Next choosing $\eta \approx 0.223$ so that $a_{0} \approx 0.223$ and $b_{0} \approx 1.397$ one finds that the mixings in (42) and (43) are satisfied.

Other values of $a, b, c, D$, and $x$ in this general range worked as well. However in general the various relationships worked best when $b$ was close to 4 .

\section{SUMMARY AND CONCLUSIONS}

In this paper we studied the field equations of fermions in the background of the nonsingular, $6 D$ brane solution of $[19,13]$. By allowing the exponent, $b$, in the $4 D$ scale function, $\phi(r)$, to take values $b>2$ we found that we could get multiple zero-mass modes which were identified with different fermion generations. In particular for $4<$ $b \leq 8$ we obtained three zero-mass modes corresponding to different $l$ eigenvalues: $l=-1,0,1$. The charge $l$ played the role of the family number. When one fixes the value of $b=2$ as in [19] one has only one zero mode [21]. For $b>2$ the 2D scale function, $\lambda$ has a zero both at $r=0$ and $r=\infty$. However the scalar invariants such as the Ricci scalar are well behaved and nonsingular over the entire range of $r$, indicating these points are coordinate rather than physical singularities.

The masses and mixings between the different generations was given by a common mechanism - the introduction of a scalar field with a Yukawa coupling to the fermions.

An interesting extension of the above scenario is to see if the scalar field could play a dual role: (i) as the mechanism for generating the masses and mixings and (ii) as the matter source for forming the brane. In $[23,24]$ it was shown that a scalar field could be used as a source to construct a thick brane. Thus it might be possible to replace the phenomenological matter sources, $F(r)$ and $K(r)$, by a scalar field source. Such a scenario would be more economical since the scalar would serve the dual role of forming the brane and giving masses and mixings to the fermions.

\section{ACKNOWLEDGMENTS}

D. S. acknowledges the CSU Fresno College of Science and Mathematics for a sabbatical leave during the period when part of this work was completed. D. S. also thanks Professor Vitaly Melnikov for the invitation to work at VNIIMS and the People's Friendship University of Russia where part of this work was completed.
[1] S. L. Glashow, Nucl. Phys. 22, 579 (1961); S. Weinberg, Phys. Rev. Lett. 19, 1264 (1967); A. Salam, in Elementary Particle Theory: Relativistic Groups and Analiticity, Nobel Symposium Vol. 8, edited by N. Svartholm (Almquist and Wiksell, Stockholm, 1968), p. 367.

[2] C. D. Froggatt and H. B. Nielsen, Nucl. Phys. B147, 277 (1979).

[3] N. Arkani-Hamed, S. Dimopoulos, and G. Dvali, Phys. Lett. B 429, 263 (1998); I. Antoniadis, N. Arkani-Hamed, S. Dimopoulos, and G. Dvali, Phys. Lett. B 436, 257 (1998).

[4] L. Randall and R. Sundrum, Phys. Rev. Lett. 83, 3370 (1999); 83, 4690 (1999).

[5] M. Gogberashvili, Int. J. Mod. Phys. D 11, 1639 (2002); 11, 1635 (2002).

[6] K. Akama, in Proceedings of the Symposium on Gauge Theory and Gravitation, Nara, Japan, edited by $\mathrm{K}$. Kikkawa, N. Nakanishi, and H. Nariai (Springer-Verlag, Berlin, 1983).
[7] V. A. Rubakov and M. E. Shaposhnikov, Phys. Lett. B 125, 136 (1983); 125, 139 (1983).

[8] M. Visser, Phys. Lett. B 159, 22 (1985).

[9] G. W. Gibbons and D. L. Wiltshire, Nucl. Phys. B287, 717 (1987).

[10] D. Cremades, L.E. Ibanez, and F. Marchesano, Nucl. Phys. B643, 93 (2002); C. Kokorelis, Nucl. Phys. B677, 115 (2004).

[11] N. Arkani-Hamed and M. Schmaltz, Phys. Rev. D 61, 033005 (2000); E. A. Mirabelli and M. Schmaltz, Phys. Rev. D 61, 113011 (2000); C. Biggio, F. Feruglio, I. Masina, and M. Perez-Victoria, Nucl. Phys. B677, 451 (2004); R. Erdem, Eur. J. Phys. C 25, 623 (2005).

[12] A. Neronov, Phys. Rev. D 65, 044004 (2002).

[13] P. Midodashvili, hep-th/0308051.

[14] G. R. Dvali and M. A. Shifman, Phys. Lett. B 475, 295 (2000); D. E. Kaplan and T. M. Tait, J. High Energy Phys. 11 (2001) 051; Z. Surujon, Phys. Rev. D 73, 016008 (2006). 
[15] C. Deffayet, G. Dvali, and G. Gabadadze, Phys. Rev. D 65, 044023 (2002); C. Deffayet et al., Phys. Rev. D 66, 024019 (2002); V. Sahni and Yu. V. Shtanov, J. Cosmol. Astropart. Phys. 11 (2003) 014.

[16] M. Gogberashvili and M. Maziashvili, Gen. Relativ. Gravit. 37, 1129 (2005); M. Gogberashvili, gr-qc/ 0511039.

[17] V. N. Melnikov, Cosmology and Gravitation, edited by M. Novello (Editions Frontieres, Singapore, 1994), p. 147; Cosmology and Gravitation II, edited by M. Novello (Editions Frontieres, Singapore, 1996), p. 465.

[18] V.D. Ivashchuk and V. N. Melnikov, Classical Quantum Gravity 18, R82 (2001).

[19] M. Gogberashvili and D. Singleton, Phys. Lett. B 582, 95 (2004).

[20] M. Gogberashvili and D. Singleton, Phys. Rev. D 69, 026004 (2004).
[21] M. Maziashvili, Phys. Lett. B 596, 311 (2004).

[22] I. Navarro and J. Santiago, J. High Energy Phys. 02 (2005) 007.

[23] S. T. Abdyrakhmanov, K. A. Bronnikov, and B.E. Meierovich, Gravitation Cosmol. 11, 82 (2005); K. A. Bronnikov and B. E. Meierovich, JETP 101, 1036 (2005).

[24] K. A. Bronnikov and B.E. Meierovich, Gravitation Cosmol. 9, 313 (2003); K. A. Bronnikov, B.E. Meierovich, and E. R. Podolyak, JETP 95, 392 (2002); K. A. Bronnikov and B. E. Meierovich, JETP 97, 1 (2003).

[25] S. Carroll and M. Guica, hep-th/0302067.

[26] P. Q. Hung and M. Seco, Nucl. Phys. B653, 123 (2003); P. Q. Hung, M. Seco, and A. Soddu, Nucl. Phys. B692, 83 (2004).

[27] A. Neronov, Phys. Rev. D 64, 044018 (2001).

[28] D. Singleton, Phys. Rev. D 70, 065013 (2004).

[29] S. Eidelman et al., Phys. Lett. B 592, 1 (2004). 